\title{
Artifacts' Roaming Beats Recognition for Estimating Care Activities in a Nursing Home
}

\author{
Francisco E. Martinez-Perez \\ Facultad de Ingeniería \\ Universidad Autónoma de Baja \\ California \\ Ensenada, México \\ fmartinezperez@acm.org
}

\author{
J. Angel Gonzalez-Fraga \\ Facultad de Ciencias \\ Universidad Autónoma de Baja \\ California \\ Ensenada, México \\ angel_fraga@uabc.edu.mx
}

\author{
Monica Tentori \\ Department of Informatics, UCI, \\ Irvine, USA \\ Facultad de Ciencias, UABC, \\ Ensenada, México \\ mtentori@uabc.mx
}

\begin{abstract}
This paper describes an approach to estimate the activity being performed by caregivers in a nursing home. The approach is based on information gathered from a workplace study conducted in a nursing home, in which 400 hours of detailed observation caregivers' activities were recorded. Automatic identification of objects based in composite correlation filters was implemented in order to generate a descriptive behavior of objects. When the behavior of the artifacts related with the activities is validated, the estimation of an activity is found. The activities estimated with a $92.72 \%$ of accuracy (on average) include hygiene, feeding and taking vital signs.
\end{abstract}

Keywords-: Activity estimation, context-aware computing, object recognition, correlation filters, artifacts' roaming beats

\section{INTRODUCTION}

The World Health Organization (WHO) indicates that 1-4\% of the world population may have some level of Intellectual Disability (ID) [1]. According to this organization:

Intellectual disability (ID) is a disorder defined by the presence of incomplete or arrested mental development, principally characterized by the deterioration of concrete functions at each stage of development and that contribute to the overall level of intelligence.

The Alzheimer's disease (AD) is considered by many as the fastest growing intellectual disability, worldwide, 30 million of people have $\mathrm{AD}$ [2]. Along with this disease elders also may present other health constraints such as restricted mobility. Certainly, caring for elders with restricted mobility on top of $\mathrm{AD}$ is a time-consuming responsibility which often imposes to caregivers more adverse consequences such as employment complications, less time for their own leisure activities and physical and emotional stress. The challenges associated with the care of elders can be overwhelming, leading caregivers to place their relatives into assisted living facilities or hire services provided by specialized clinics.

Specialized caregivers at these homes or clinics need to monitor the behavioral patterns of elders with restricted mobility to record disease progress, identify abuse and make decisions about whether a treatment is working. In particular, ubiquitous computing technologies can enable the capture of and access to real time video in these environments to monitor

Identify applicable sponsor/s here. (sponsors) those events of interest that arose in the care of people with ID. The goal of ubiquitous computing is to enhance computer use by making many computers available throughout the physical environment which are effectively invisible to the user [3]. By many available ubiquitous technologies, capture and access tools $[4,5,6]$ are particularly promising for monitoring the effectiveness of interventions. They are

tools to support the automated capture of and access to live experiences aimed at augmenting the inefficiency of human record-taking, especially when there are multiple streams of related information that are virtually impossible to capture them manually [5]

With such tools health and behavioral data can be captured, analyzed, and mined over time providing valuable evidence for care delivery.

The design of such a tool faces one major challenge: it is important to identify which information is worth capturing and how these moments of interest could be automatically detected. For example, reviewing videos captured 24 hours a day 7 days a week is a time consuming task requiring an enormous amount of storage capacity. Therefore, we must develop mechanisms that allow the automatic recognition of relevant events to be archived and indexed.

While considerable work has been done in the development of robust approaches to estimate the time, location and identity dimensions of context, the activity dimension has been left aside. Since human activities are complex and dynamic, it is often unclear what information is relevant for modeling activities and how an activity can be estimated. Hence, robust approaches to estimate human activities must be developed along with the technology necessary for such estimation. These approaches must establish which information is relevant to infer an activity, how much automatic sensing is needed and which technologies are appropriate to sense such information.

In this paper we discuss an approach for the estimation of activities taking into account the recognition of the roaming beats of artifacts. This approach is based on the use of compose correlation filters to recognize objects' roaming beats. The filter is trained and evaluated with data captured from close to 400 hours of detailed observation and documentation of patients and caregivers at a nursing home. The rest of this paper 


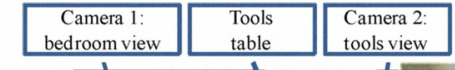

a)

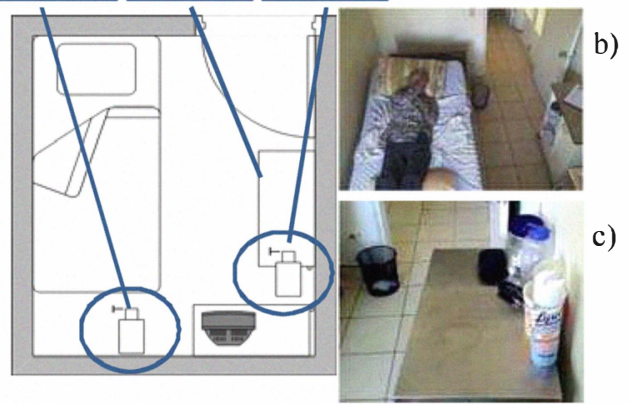

Figure 1. (a) Position of the cameras in each patient room (b) A patient recorded with the camera with room view (c) The tools table recorded with the camera with tools view

is organized as follows. In Section 2, we discuss the results of a case study conducted with the aim at understanding the care of elders with restricted mobility. Section 3 discuss our approach for artifacts' roaming beats and activity recognition. In Section 4 , we present the results of the approach. Finally, Section 5 presents the conclusions and directions for future work.

\section{A. Background-Artifacts recognition for activity estimation}

Several efforts have been made to detect the artifacts used by a user and then use this information to infer activities. These approaches range from attempts that demand from the user to wear complex equipment to methods that combine the advantage of easy sensor deployment with that of unobtrusive sensor detection. For instance, the RFID-detecting glove housed in a small box a SkyeTek RFID reader which communicates to an antenna the information captured [7]. Hence, the glove acts as an information proxy between the user and the artifacts with RFID tags attached. Thus, each time a user grabs a tagged artifact the glove will capture such information identifying the artifact being used at that particular moment. Another approach in this direction are sensors designed as "tape on and forget" devices that can be quickly and ubiquitously installed in real settings. In this approach, "stick-on" sensors with an accelerometer, clock, and local memory are placed on the objects [8]. Accelerometers detect touching, and record that to local memory. When the sensors are later removed, the data can be analyzed to reconstruct the set of all object touches. Alternatively, the two types of sensors described above can be combined, by using the accelerometer from the first type of sensor to detect interactions and the RF protocol of the second one, to transmit this information wirelessly. This method has potential, but has the disadvantage of being incompatible with the commercial tags. Hence, techniques that operate by interacting with long-range readers have been proposed [9]. However, knowing that a user is holding a medical record does not provide enough evidence to judge if a user is engaged in information management or clinical case assessment. By knowing that the hospital worker uses a typewriter along with the medical record, our approach could infer that such user is performing activities related to information management. Hence, combining measures from multiple sensors could increase the confidence value for a particular interpretation. This approach requires saturating the hospital premises with pervasive sensors and technology that must assemble and transmit contextual information from different sources in the environment, resulting thus, in a wireless sensor network. This sensor network must have capabilities for context fusion providing reliable ubiquitous context by combining services in parallel (to offset noise in the signal) and sequentially (to provide greater coverage) [10]

\section{A CASE STUDY IN A NURSING HOME: UNDERSTANDING THE CARE OF ELDERS WITH RESTRICTED MOBILITY}

We conducted a case study with the aim at: (1) to understand the care of elders' with restricted mobility, (2) to characterize the care activities executed in a nursing home and (3) to identify the purpose of object usage when performing care activities. The study was conducted in a private nursing home in the city of Ensenada, Mexico. This institution mainly focuses in the care of elders with Alzheimer and Senile Dementia and it also provide daycare services for elders who are recovering from a surgery or suffering a terminal disease. Seven caregivers rotating shifts of 8 hours attend 15 elders: 7 with Alzheimer, 1 with Senile Dementia, 1 with Parkinson and 1 recovering from a femur fracture.

\section{A. Methods}

The case study was conducted for a period of four months where two patients living in the nursing home and with restricted mobility and their caregivers were shadowed for three complete working shifts and interviewed by a couple of researchers. Each subject was introduced to the study, and was asked to participate voluntarily (Figure 1).

As Figure 1 illustrates, the observational study was video recorded with two cameras: one located in the ceiling of the room (Figure 1b) and the other located in the tools table (Figure 1c). We obtained close to 400 hours of video with an average per day of 30 hours.

To analyze the data collected we used grounded theory techniques [11]. This qualitative analysis helped us to create a coding scheme that shows the artifacts used, the people involved in and the activities executed when caring elders with restricted mobility. Then we measured the time spent by hospital workers performing different activities, the average number of activity segments and the mean time of activity segments observed for each subject. Each segment accounts for the uninterrupted engagement in a particular behavior.

\section{B. Results and discussion}

As a result of analysis we identified the type of the activities executed by caregivers when caring elders with restricted mobility, the behavior of the objects used when performing such activities as well as the strategies followed and problems faced.

1) Activities performed to care elders with restricted mobility in a nursing home

Figure 2, illustrates a scenario that describes a typical working day of one of the caregivers observed highlighting the activities executed, the routines of daily care [12] and artifacts usage.

"Mrs. Aurora is a 65 years old woman suffering of overweight and diabetes. She is immobile due to an accident and ever since, her skin is constantly filled with sores. The sores in her skin appear because she is immobile and nobody 
in her family can help her to perform the exercises suggested by the physician. Their relatives concerned with her health problems decided to place her into a nursing home where specialized caregivers could closely monitor her health and help her to fulfill her basic needs. At the nursing home, the caregiver Juan elaborates a care plan for Mrs. Aurora including therapies, medicine and food administration, among others. At 8:00 am when Juan arrives he moves to Mrs. Aurora's room and helps her to eat her breakfast and administers her medicines. To this aim, Juan placed in the tools table the food tray and the medicines dispenser. While helping Mrs. Aurora to eat her breakfast he takes from the tools table the artifacts needed for the adequate execution of the activities. After, Juan placed the vital signs kit on the tools table, then, he takes her vital signs and her level of glucose. At noon, while Mrs. Aurora's talks with her son who came to visit her, Juan realizes that the sore on Mrs. Aurora's arm is getting worse. Juan moves to Mrs. Aurora's room and baths her while treating her sore. Once again he places the hygiene tools on the tools table. For one hour, Juan moves the hygiene artifacts from the tools table to Aurora's bed back and forth. Finally, Juan starts his routine again by taking Mrs. Aurora vital signs, helping her with her meals and performing her therapies. After two weeks Mrs. Aurora's sores start to vanish and her health improves significantly"

This scenario illustrates caregivers' responsibilities and the activities performed to improve elders' quality of life. As it shows, we identified seven care activities: medicines administration, meals ingesting, health monitoring, visit, clinical evidence management, preparation and therapies. We found that caregivers spent most of their time during preparation (23.03\%), following by health monitoring $(19.69 \%)$ and finally by hygiene (17.22\%). However, during health monitoring, caregivers monitor the quality when performing medicine administration, meals ingesting, hygiene and motion therapies

2) Artifacts' role in monitoring care activities

As the scenario illustrates one of the most important activities of caregivers is to monitor elders' health and the artifacts role when monitoring care activities. As one caregiver explained:

"it is extremely important to have visual evidence of the activities executed by caregivers [and patients] because [a mistake] could cause a negative impact in elders' health"

Thus, caregivers may need to consult two types of activity awareness: (1) a history of past behavior and (2) the activities executed by an elder during a day. In this regard, the caregiver's manager mentioned that he was interested in keeping a record of elder's activities in part because elders' relatives do frequently request such information. To this aim, caregivers currently maintain an activity log were they register the actions they performed for each patient. For instance, every time an activity is executed, the caregiver makes a new entry in the log specifying contextual information associated to such activity such as location, time of the day or description. Despite of this some inconsistencies may arise when maintaining and consulting the log. For instance, caregivers explained that several times they forget to update the log after executing an activity. To this aim one strategy followed by caregivers is to infer logs entries based on artifacts behavior. For instance, if no entry related with the hygiene of a patient is reported but the hygiene artifacts are on the tools' table or in the trash, the activity was probably executed but not reported in the log. In contrast, if the activity of "give medicines" has been reported but the medicine dispenser has not moved from the medicine cabinet such medicine may not be administered. When a situation like this one occurs caregivers ask others about the inconsistency and most of the time the error is fixed.

Based on caregivers' strategy of monitoring artifacts behavior we analyze the artifacts usage when performing care activities. We found out that for each activity the artifacts have a "base location" were they are manipulated and each follows what we call a "roaming beat" -in the scenario the base location of the artifacts being used is the "tools table". We define a roaming beat as the ability of an artifact to given a time stamp shift from:

- a motionless to a mobile state and,

- from a base location to a mobile one

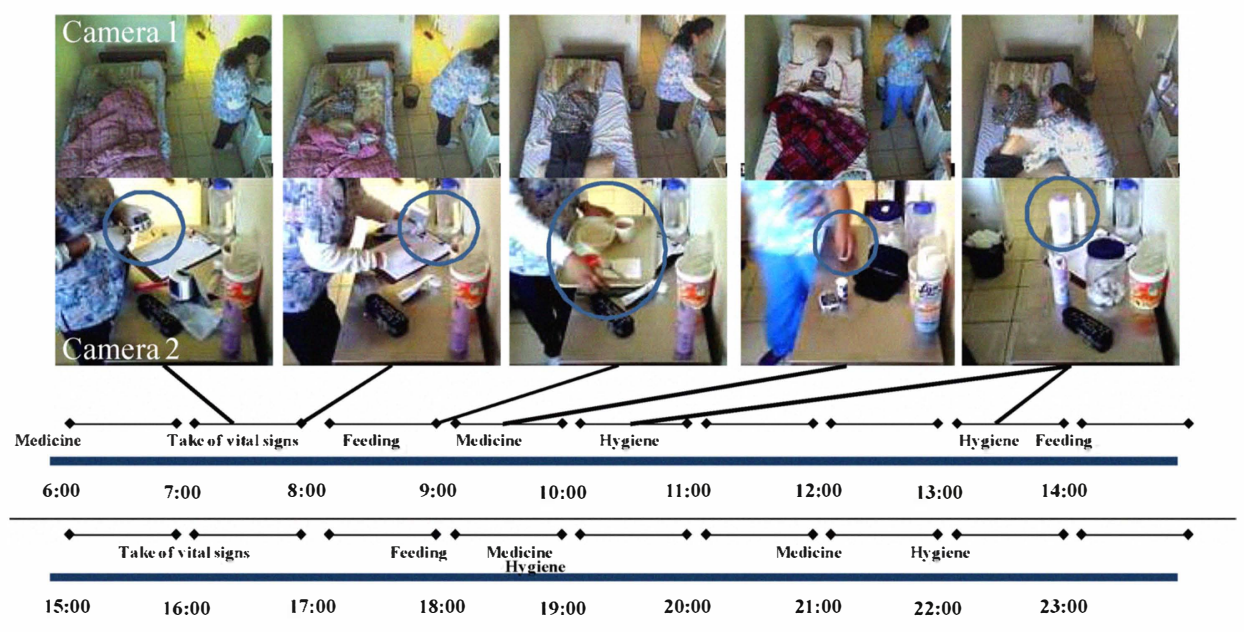

Figure 2. Care activities performed by a caregiver during a working day of observation 
For instance, as described in the scenario when Juan was performing the activity of hygiene by moving back and forth hygiene tools. A beat acts as a motion marker determining the state of an artifact. For instance, in the scenario when the medicine dispenser is removed from the tools view. Finally, we observed that an artifact could live in three states:

- Initialized, when an artifact it is being set up and placed in its base location (e.g., the tools table).

- $\quad$ activated, when an artifact's beat change (e.g., from a motionless to a mobile state) or remain in the same state (e.g., mobile) and;

- $\quad$ suspended, when an artifact remains motionless and it is suspended

For instance, the following scenario illustrates the roaming beat of the vital signs kit (i.e., kit bag) when the caregiver Juan took the vital signs of Mrs. Aurora:

Kit bag [initialized - first beat]: The kit bag relies immobile on the tools table.

Kit bag [activated - second beat]: Juan grabs the thermometer from the kit and moves it to Mrs. Aurora's bed (i.e., mobile location) to take her temperature. The kit bag is mobile.

Kit bag [activated - third beat]: Juan switches the thermometer for the glucose meter to take Mrs. Aurora level of glucose. The kit bag remains mobile.

Kit bag [suspended-last beat]: Juan leaves the kit bag and it became immobile

By observing this artifacts' roaming beats we found out that the activity being performed by a caregiver could be estimated by determining artifact's beats and their state.

\section{VISION-BASED TECHNIQUES FOR ARTIFACTS' ROAMING BEATS AND ACTIVITY RECOGNITION}

Based on the hypothesis that activities may be inferred by the interactions with objects $[13,7]$ and artifacts' roaming beats we first propose an approach for object recognition to then use this information for activity estimation. In the following lines we describe our method in detail:

\section{A. Object recognition}

We decided to use compose correlation filters to estimate artifacts' roaming beats. Object recognition based on correlation filters computes a level of similarity between two references signals that for our case were images: (1) the reference image and (2) the target image or the workplace scene. The image of the workplace scene is used to test the filter in real time and it is matched with a reference image previously recorded and used to train the filter. For instance, for our case our reference image is a scene recorded during our case study and the workplace scene is the one to be taken when the caregiver is caring for the elder at the nursing home. One of the advantages in using correlation filters is that we can locate in a scene multiple objects without segmentation reducing the time of processing.
We analyzed the data gathered from the camera recording the tools table (Figure 1c) and based on such information we inferred the type of activity being portrayed in the scenes recorded by the camera with the bedroom view (Figure 1b) -to avoid inconsistencies between the cameras they were synchronized. For recording the video we used two Linksys wvc53gca cameras capturing video in MPEG 4 format using a resolution of $320 \times 240$ pixels. Videos did not suffer any modification with respect the introduction of segmentation marks. We generated 4 minute segments to facilitate the qualitative analysis and the automatically objects' search. We used MatLab to implement the filters.

In image processing, object recognition it is not a simple task. For some applications we need to take into account environmental noise and object characteristics. For instance, objects have three intrinsic features including their form, size and color that sometimes may be confused with other objects or such characteristics may be distorted. For instance, objects may be rotated, escalated or deformed based on the proximity with the camera when the picture was taken and based on the illumination and shadow conditions. Based on the results of our case study we identified the objects that are used when conducting care activities. Table 1 shows the objects used to recognize care activities or actions. As it shows, we used two types of correlation filters for: (1) vision-based tags recognition and (2) features-based object recognition.

\section{1) Vision-based tags recognition}

Recognizing objects based on their features is very difficult because some objects have similar features. For instance, the size and shape of a bottle of water it is the same as an alcohol bottle. Thus we decided to use a tag to differentiate those objects with similar features. By doing so the correlation filter will be able to detect the tag and then infer the object associated to such tag.

To use this method the big challenge is to select a tag that will be somehow differing from the others tags used within a scene (Figure 3a). To design our tags we used a dissimilarity function to guarantee that the tags used in one scene will be different. Then, we conducted several experiments following the techniques proposed in $[9,10]$ to make minor adjustments to

Table 1. Vision-based approach used for object recognition

\begin{tabular}{|c|c|c|c|}
\hline \multirow{2}{*}{ Kind of activity } & \multirow{2}{*}{ Object } & \multicolumn{2}{|c|}{ Recognition method } \\
\cline { 3 - 4 } & & Features & Labeling \\
\hline Hygiene of elderly & Paper towel & Yes & No \\
\hline & Cream oil & No & Yes \\
\hline & Diaper & Yes & No \\
\hline & $\begin{array}{c}\text { Physiological } \\
\text { solution }\end{array}$ & No & Yes \\
\hline & Talc & No & Yes \\
\hline & Billbox & No & Yes \\
\hline Take medicine & Plate & Yes & No \\
\hline Foods & Glass of water & Yes & No \\
\hline & Cup & Yes & No \\
\hline & Charola & Yes & No \\
\hline Vital signs (Pressure) & Baumanometer & No & Yes \\
\hline Vital signs (Dextrose) & Kit Bag & No & Yes \\
\hline
\end{tabular}




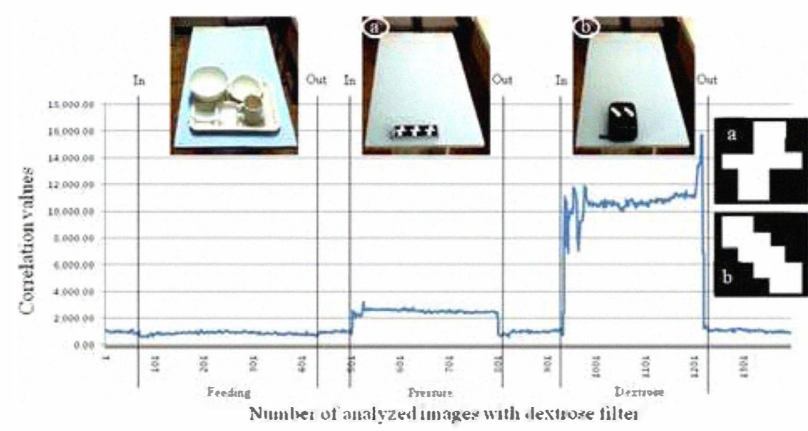

Figure 3. Two vision-based tags used to recognize (1) blood pressure label (2) dextrose label

our dissimilarity function. Table 1 shows the objects tagged.

\section{2) Features-based object recognition}

In contrast, the features of other objects differ significantly and also it is impossible to use a tag for its recognition. For instance, the toilet paper changes its appearance every time it is used. Thus, as table 1 show, we decided to use another filter to recognize certain objects taking into account their features.

For both cases we created a correlation filter that by identifying either tags or objects' features recognizes one object in a scene -for recognizing multiple objects we implemented as many filters as objects to be recognized. To synthesize each filter we used three images of the object or of the tag at different distances, from the bedroom view and the tools table view -cameras were in the same position as used during the case study. Then we took into account the image segmented diffracting among the top, bottom and center parts of the image. We finally tested the filters separately by capturing the images directly from the video camera.

Figure 3 shows our filter behavior designed for the identification of the dextrose kit used when measuring an elder's level of glucose. The x-axis shows the number of frames analyzed and the $y$-axis shows the correlation values result for our experiments. To test the filter within the lab we introduced three images that have different features and tags at different time intervals (i.e. frames). The video starts without objects in the scene and in frame number 70 the food tray is introduced. As Figure 3 shows, the filter does not reflect difference in the correlation values. Then, in frame 500 the pressure label is introduced. As the Figure shows, the correlation values slightly increased indicating a slight similarity between the reference images (used to train the filter) and the target images (used to test the filter). Finally, in frame 920 the dextrose kit its introduced and the correlation values dramatically increased. This indicates a strong similarity between the reference and the target images after a threshold. For each object in Table 1 a similar procedure was followed for object recognition.

\section{B. Inferring activity from recognized objects' roaming beats}

With our object recognition approach we are capable of determining objects' roaming beats by noticing when each object is moved from its "base location" to a mobile one or switching among motion states. For instance, consider the following example taken from the scenario described in section 2:

Baumanometer [initialized -first beat]: The caregiver Juan places the baumanometer in the tools table

Baumanometer [activated - second beat]: Juan moves to Mrs. Aurora's bed and unwraps her arm. The baumanometer remains in the tools table.

Baumanometer [activated - third beat]: Juan grabs the baumanometer and places it around Mrs. Aurora's arm away from the tools table.

Baumanometer [activated - fourth beat]: After a while, Juan removes the baumanometer and places in the tools table. He registers the activity in the activity log.

Baumanometer [suspended - last beat]: Finally, Juan removes the baumanometer from the tools table.

As the scenario shows three pattern behaviors are exhibited in the use of artifacts. In the following lines we explain each one and how they are used for activity estimation:

- An artifact is initialized with the first beat. The first beat of an artifact occurs whenever:

i. a new artifact is placed in a base location, for instance, when the baumanometer is first placed on the tools table;

ii. an immobile artifact switches to a mobile state, for instance, when Juan grabs the thermometer from the kit bag and;

iii. one of the above conditions is met and a shift in the activity occurs, for instance, when Juan switches from food ingestion to medicine administration by grabbing

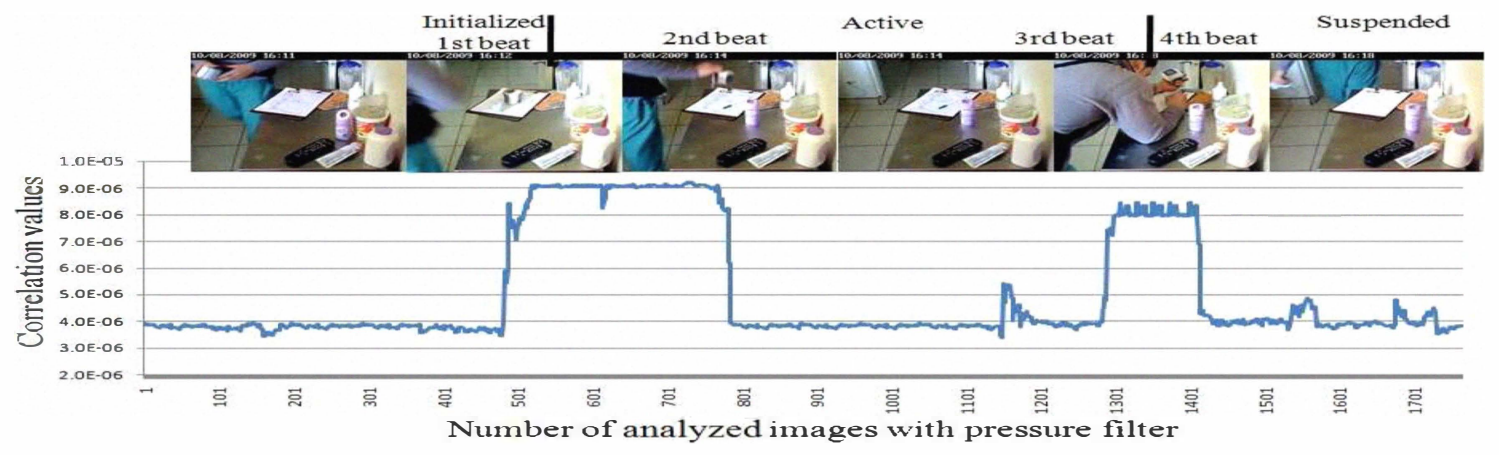

Figure 4. Using the pressure filter for recognizing roaming bits of the baumanometer and then inferring the activity of "taking pressure" 
the medicines dispenser and leaving the food tray.

The first beat of an artifact could be used to set a beginning marker specifying that a new activity is detected or newly recognized.

- An artifact is activated and sequential beats present a recurrence phenomenon. After the first beat is detected the behavior pattern in artifacts usage changes from an immobile state to a mobile one. These sequential beats could be detected when:

i. the number of times an artifact is being used surpasses a threshold, for instance, when the baumanometer is used two times and;

ii. the time an artifact spends in its mobile location and returns to its base location by moving back and forth surpasses a threshold, for instance, when the baumanometer is removed from the tools table to Mrs. Aurora's arm and then placed again the tools table when Juan is registering the activity in the log.

- An artifact is suspended after it last beat. An activity has concluded when an artifact:

i. remains immobile, for instance, when the kit bag was left immobile on the tools table and;

ii. is away from its base location, for instance, when Juan removes the baumanometer from Mrs. Aurora's room.

For activity recognition we must be able to detect the artifacts' roaming beats and then infer each artifact state. In other words, we must identify how artifacts are being manipulated when performing care activities. To this aim, we used the information gathered in our case study to define thresholds and intervals for each object used when performing a specific activity. Figure 4 shows an example of how the roaming beats of the baumanometer artifact were recognized. Going back to our example:

Baumanometer [no beats]: The video starts when Juan arrives to Mrs. Aurora's room.

Baumanometer [initialized - first beat]: At frame 480 the correlation values dramatically increased recognizing the first beat of the baumanometer when it is placed in the table.

Baumanometer [activated - second beat]: From frame 480 to frame 775 the correlation values remain steady indicating that the baumanometer is immobile on the table.

Baumanometer [activated - third beat]: At frame 776 the correlation values dramatically decreased indicating that the baumanometer has been removed from the table. During this period of time Juan is taking the vital signs of Mrs. Aurora.

Baumanometer [activated-four beat]: At frame 1290 the correlation values increase again indicating the baumanometer's presence.

Baumanometer [activated - fifth beat]: From frame 1290 to frame 1411 the correlation values remain steady indicating that the baumanometer is immobile on the table while Juan registers the activity in the log.
Baumanometer [suspended - last beat]: Finally, at frame 1411 Juan removes the baumanometer from the tools table and after a threshold no activity is detected with such artifact.

Note that around Frames 1101 and 1501 the correlation values slightly increase indicating a slightly confusion with other objects that appear on the scene.

\section{Limitations of our approach}

Although the composite correlation filters we implemented are capable of recognizing objects with a degree of scale and when they are in different positions, we did not take into account other types of noise or problems that could be presented when taking the images such as, environment illumination, rotated objects or object intrusion. Similarly, the results obtained are constrained by the data used to train and test the filter. While, the data is exhaustive, it was collected in a small-size nursing home. Hence, it is not possible to derive findings that can be directly extended to other nursing homes. However, we want to clarify that our efforts centered on acquiring a vast and detailed understanding of the practices of a few individuals across a varied set of circumstances. Therefore, the significance of our results lies in the qualitative nature of the inquiry and the analytical method that we conducted.

Also, the criteria used for objects and activities recognition might not be generalizable. We based our criteria upon specific rules, as others have proposed [14], and the design of specific tags that could only be used in this context. However, our aim here was to test our method in a controlled environment to show the applicability and feasibility of our approach and then incorporate further complexity into our problem. Our rulebased approach could always be substituted with a more complex algorithm as we have done in the past $[13,15,16]$

Finally, the technique used to gather the data requires extensive field work. This is a disadvantage of the approach. However, a smaller field study could be made to tune the classifier to a new setting. In addition, we must be aware of the amount of data that we can obtain from handling video stream. It involves to the elderly, staff and relatives, but their perceptions and implications have not been considering in this research. Others topics for us are the privacy and security of data, but these are beyond our reach.

\section{RESULTS AND DISCUSSION}

\section{A. Evaluation}

In order to evaluate the system proposed, we conducted several experiments in the usability lab at UABC campus Ensenada, Mexico. We perform many simulations for each of the activities shown on table 1. Simulations were conducted in different ways, i.e. arranging and handling the objects that are used on the tools table for the performance of the activities. A camera was used to monitor all activities that were happening on the tools table. This camera was placed exactly in the same conditions as the nursing home's camera. In the lab the light conditions were controlled and this was the only one difference with the nursing home setting.

We wrote a script based on the activities behavior identified in the case study, before to start the evaluation. The evaluation was based on the script. It helped us to replicate the handling of 


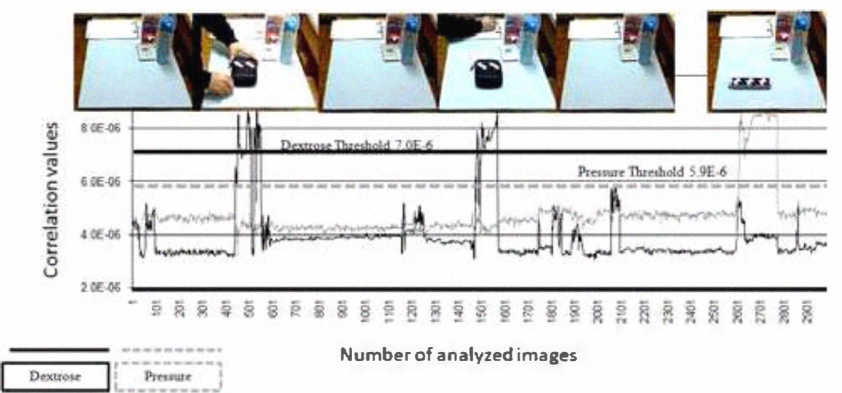

Figure 5. Recognition a dextrose activity

the objects used within the activities inside the nursing home. Objects' behavior, and start and end time of activities were considered.

The script indicated the time in which the object must enter on the scene, the number of times that it must be removed and re-entered. This simulation process was very important because we did not have to come back at the nursing home to generate the evidence. Practically, the activities were replicated under the same circumstances and conditions of the handling of the objects without affecting the activities of caregivers or elders.

The system was evaluated in the usability lab over a period of 5 days. In this evaluation, we performed 11 activities per day, established on the script; these were 4 hygiene activities, 3 feedings, 2 blood pressure measure and 2 samples of glucose (dextrose). In total, we carried out 55 activities that were recorded and processed. Our system was able to recognize 51 activities, and the rate of effectiveness for activities inference was $92.72 \%$. The missing activities that were not identified, that's because, these did not exceed the established threshold. We talk about of activities performance with the script in the next section.

\section{B. Object recognition}

The performance of the script was recorded to obtain the evaluation of our filters for the estimation criteria and the estimation of the activity. The process was developed considering three variants:

Case 1: Inserting an object, in which case, the recognizing is done through its own features. A paper towel inside the hygiene activity characteristic that it is very active, i.e., enters and leaves several times on the tools table. The values that exceed the threshold of 4.9E-6 (figure 6) indicate the correct identification of paper towel and the number of times it left and entered in the setting. Recall that the object's behavior is determined by the number of times it enters and leaves the setting. As can be seen, the performance of the filter is not affected by the insertion of other objects in the setting.

When the filter identifies the object the first time, the system marks the first beat and the start of the activity is confirmed, and it also records the time. In the case of hygiene activity shown in Figure 6, the minimum threshold (set up at 4.9E-6) has been exceeded more than 8 times, meaning that the reference object has entered and left that same number of times in the setting. This behavior of the object verifies the second beat, i.e. the continuity of the activity.

The last beat of the system counts the running time of the activity. That is it, records the time from the first appearance of the object and ends when the exit impulse is confirmed. It happens when the object is completely immobile or the object goes off of tools table.

Case 2: Inserting an object in which case, the recognizing is done through labeling an object. By means of label images, filters for recognizing the dextrose kit (artifact used for doing glucose measurements) was designed and another for recognizing the baumanometer (artifact used for doing blood pressure measurements). The solid line in figure 5 is the output of the dextrose filter and the dotted line is the output of the baumanometer filter through of a video sequence where the activities of vital signs where performed.

The inference of the glucose measurement activity was done as follows. As can be seen in figure 5, the solid line exceed twice the threshold set up at 7.0E-6, meaning that the correlation filter has found twice the object in the setting in different times. This behavior was observed in the case study and similarly, the system has found the activity. The graph shows some jumps in the correlation outputs without reaching the thresholds, these small changes correspond to the entry of other objects in the execution of another activity. This example shows how the activities are overlapping, but the objects do not exceed the threshold indicated for the filter that is in execution.

In the activity of blood pressure measurement represented by the dotted line, the baumanometer is recognized in frame 2600 and remains on the table until the frame 2800 . This event does not affect the dextrose activity estimation because as stated earlier, the processes are running independently and each process checks only their threshold. In this example, the pressure measurement activity is rejected because the system

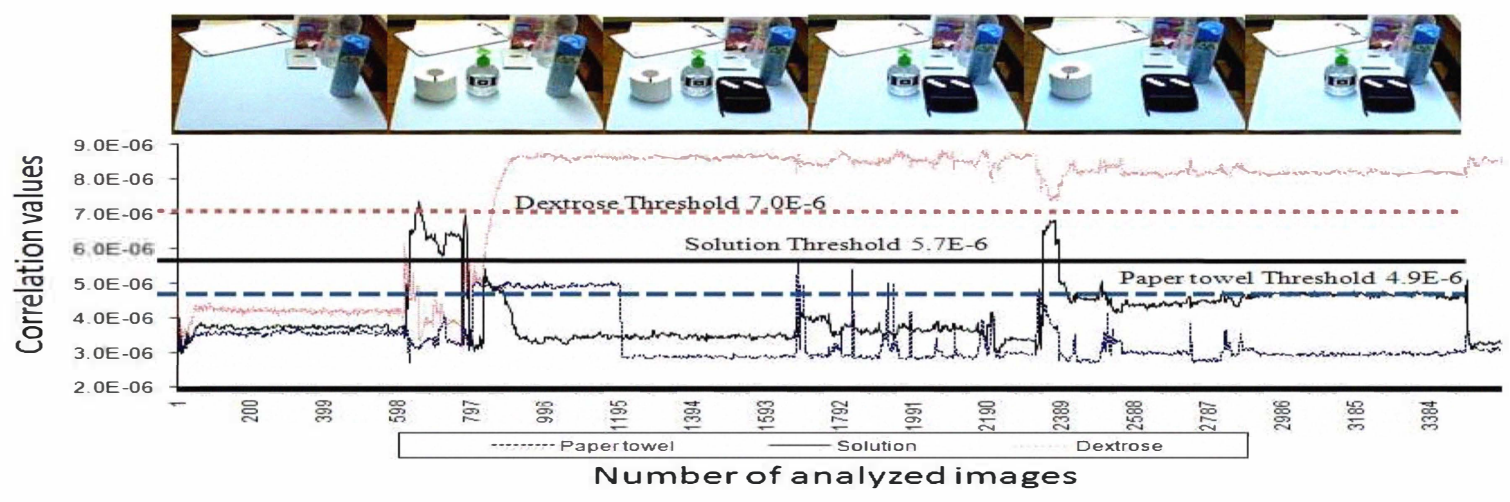

Figure 6. Recognition a hygiene activity with two objects 
indicates a start pulse of activity but does not detect the continuity in the handling of the object and hence no activity.

Case 3: Inserting several objects with mixture recognizing. In figure 6 are showed the results of three correlation filters running through the video sequence. These are executed concurrently. This situation corresponds to the hygiene activity and the recognizing process is more complex than cases 1 and 2 , because the behavior of continuity depends on several objects to estimate the activity. The activity identification holds if and only if two or more objects involved in the hygiene of elderly are handled in a span of time (see Table 1).

The paper towel in the activity of hygiene has a very active behavior. The shortest time in which it enters and leaves the table in the execution of the activity is 1 to 3 seconds. As showed in the figure 6 , their recognition appears as a narrow pulse. In the example, at the same time is running a recognition filter for the labeled object physiological solution. If both objects have a continuity handled, then activity of hygiene is confirmed.

As mentioned early, the execution of the filter is a process that must carry out the activity estimation approach proposed. In this sense, the third graph in figure 6 shows the presence of the dextrose kit but there is no activity with this artifact, so the dextrose measurement is discarded.

\section{CONCLUSIONS}

Estimating activities in an automatic way is a complex task; few attempts have been conducted to address this problem. In this paper, we presented an approach that uses composite correlation filters for the identification of objects related with a particular caring of elder activity. Each object, handled by a caregiver, has a particular behavior. The behavior of the object is strongly related with a particular activity. Hence, we proposed the inference of activities based in the behavior of the objects.

From a case study in a nursing home for older adults, we identified the main activities performed by caregivers to care older people with restricted mobility. Privacy concerns were not considered. With our approach le the automatic monitoring of some care activities is feasible.

Through the object tracking, is possible to estimate an activity. The object recognition is performed using two kinds of objects: the objects by themselves and labeling objects. We present the object recognition and estimation criteria for the activity using an analysis of the objects behavior. This behavior is presented as a train of pulses. The number of pulses and its periodicity determine the execution of an activity. Our system was able to recognize 51 activities from 55 performed, and the rate of effectiveness for activities inference was $92.72 \%$. We could increase effectiveness by adding rotation and occlusion invariance in object recognition method.

\section{ACKNOWLEDGMENT}

We thank the personnel at Lourdes Nursing Home in Ensenada, Mexico, especially to Argel Grisolle. This work was founded by PROMEP under the contract 9044 and through the scholarship provided by the first author (CONACYT 243422/217747).This work has been developed within the program Maestría y Doctorado en Ciencias e Ingeniería (MyDCI).

\section{REFERENCES}

[1] Organization, W.H., Ageing and intellectual disabilities - improving longevity and promoting healthy ageing: Summative report. Geneva, Switzerland: WHO, 2000., 2000.

[2] A newsletter for Alzheimer's Disease International, The International Federation of Alzheimer's Disease and Related Disorders Societies Inc. Global Perspective, ALZ Volume 18 No.3, December 2008.

[3] M.Weiser, Some Computer Science Problems in Ubiquitous Computing. Communications of the ACM, 1993. 36(7): p. 78-84.

[4] G.D. Abowd, , C.G. Atkenson, J. Brotherton, T. Enqvist, P. Gulley, and J. Lemon. Investigating the capture, integration and access problem of ubiquitous computing in an educational setting. in ACM Conference on Human Factors in Computing Systems (CHI '98). 1998. Los Angeles, CA, Apr. 18-23.

[5] G. D. Abowd. and E.D. Mynatt, Charting Past, Present, and Future Research in Ubiquitous Computing. ACM Transactions on ComputerHuman Interaction (TOCHI), 2000. 7(1): p. 29-58.

[6] K. N. Truong, G.D. Abowd, and J.A. Brotherton. Who, What, When, Where, How: Design Issues of Capture and Access Applications. in Ubicomp. 2001. Atlanta, Georgia.

[7] M. Philipose, K.P. Fishkin, and M. Perkowi, "Inferring Activities from Interactions with Objects",IEEE Pervasive Computing, Vol. 3, No. 4, pp. 50-57, 2004.

[8] E.T. Munguia, S.S. Intille, and K. Larson, "Activity Recognition in the Home Using Simple and Ubiquitous Sensors", In Proc. of the Pervasive, 2004, pp.

[9] K.P. Fishkin, B. Jiang, M. Philipose, and S. Roy, "I Sense a Disturbance in the Force: Unobtrusive Detection of Interactions with RFID-tagged Objects", In Proc. of the Ubicomp, 2004, pp.

[10] G. Abowd and E. Mynatt, "The Humen Experience",IEEE Pervasive, Vol. 1, No. 1, pp. 48-57, 2002.

[11] A. Strauss and J. Corbin, "Basics of Qualitative Research: Techniques and procedures for developing grounded theory", Thousand Oaks, CA., 1998

[12] Tentori, M., Rodriguez, M. D. and Favela, J. "An agent-based middleware for the design of activity-aware applications" Accepted for publication in IEEE Intelligent Systems. 2010

[13] Favela, J., M. Tentori, L. Castro, V. Gonzalez, E. Moran, and A.I. Martinez. Estimating Hospital Workers' Activities and its use in Context-Aware Hospital Applications. in Pervasive Healthcare. 2006. Salzburg, Austria.

[14] JE Bardram Applications of Context-Aware Computing in Hospital Work - Examples and Design Principles. Computer. 2004:1574-1579.

[15] J. Favela, M. Tentori, L.A. Castro, V.M. Gonzalez, E.B. Moran, and A.I. Martínez-García, Activity Recognition for Context-Aware Hospital Applications: Issues and Opportunities for the Deployment of Pervasive Networks. Accepted for publication in MONET, 2007.

[16] D. Sanchez, M. Tentori, and J. Favela. Hidden Markov Models for Activity Recognition in Ambient Intelligence Environments. in ENC. 2007. Morelia, Michocan. 